\title{
Metabolic characteristics of immature myocardium
}

\author{
Fumio Yamamoto, MD
}

Received: 17 July 2009

(C) The Japanese Association for Thoracic Surgery 2010

Myocardial protection has been recognized to have contributed enormously to the improvement of operative results in cardiac surgery, which has been attributed to basic and clinical research aimed at better protective methods in many medical institutions since 1970s. Especially, postoperative outcomes of adult cardiac surgery have recently improved and become stable. In March 2007, the Japanese Meeting of Research for Myocardial Protection, a nationwide annual meeting aimed at improving surgical myocardial protection, was dissolved so a better organization might be formed in an attempt at collaboration among various research fields. There was a need for such collaboration because heart research regarding myocardial protection has become methodologically specialized and recently involved in a field of regeneration medicine that may be technically difficult for clinicians such as cardiovascular surgeons.

Historically, around the world, surgical myocardial protection in adults has been extensively studied, whereas in the field of congenital cardiac surgery (with a higher postoperative mortality compared to adult cardiac surgery) there is no established method for surgical myocardial protection, reflected in lower research activity in terms of immature myocardium. A discrepancy in myocardial protection during open heart surgery between adults and children has been considered to depend on the differences in myocardial characteristics in various

This editorial refers to the article by Fujii et al. on pp. 174-181 of this issue of General Thoracic and Cardiovascular Surgery.

F. Yamamoto $(\bowtie)$

Department of Cardiovascular Surgery, Akita University School of Medicine, Hondo 1-1-1 Akita, Akita 010-8543, Japan

Tel. +81-18-884-6135; Fax +81-18-836-2625

e-mail: f-yama@cvs.med.akita-u.ac.jp aspects. This background difference between the mature and immature myocardium may raise a question: "Would it be possible to use a cardioplegic solution for mature hearts in congenital heart surgery?" Especially, the influence of long-term preexisting cyanosis after birth on the protective effect of a cardioplegic solution should be investigated in detail to establish a method of myocardial protection for congenital heart surgery.

Is there any difference in the physiological and biochemical aspects between the mature and immature myocardium? Doenst and Taegtmeyer widely reviewed studies dealing with immature myocardium and reported in detail the difference between the mature and immature myocardium in terms of the effects of energy substrate utilization on ischemic preconditioning. ${ }^{1}$ In 1983 , Rolph and Jones, in their study using guinea pigs, reported that energy-producing metabolism in the immature myocardium is more dependent on glycolysis than that in the mature myocardium. ${ }^{2}$ In 1991, Lopaschuk et al. concluded that in human myocardium glycolysis is the predominant source of ATP production immediately after birth. ${ }^{3}$ Grosso et al. reported in 1992 that less 5'nucleotidase activity in the immature myocardium is associated with more preservation of high-energy phosphate. ${ }^{4}$ Bolling et al. demonstrated in 1992 that it resulted in enhanced myocardial protection, ${ }^{5}$ and Pridjian et al. reported in 1994 that the cardioprotective effect of inhibiting $5^{\prime}$-nucleotidase activity may have developmental changes. ${ }^{6}$

Myocardium produces ATP and uses it as an energy source. Energy substrates for ATP production include fatty acid, glucose, and lactate. Fatty acid is a main energy substrate in the mature myocardium, whereas glucose is the main one in immature myocardium. This difference is known to be caused by differences in the 
mechanisms that produce ATP in the mature and immature myocardium: Mature myocardium has well-developed mitochondria producing high-energy phosphate aerobically through the TCA cycle and oxidative phosphorylation, whereas immature myocardium, having underdeveloped mitochondria, has abundant intracellular glycogen granules, reflecting recruitment of glycogen as an energy substrate of anaerobic ATP production. Glycolysis-dependent ATP production can function under the condition of a low oxygen supply, which means that immature myocardium has an advantage over mature myocardium under hypoxia, causing greater tolerance to hypoxic or ischemic insult in the immature myocardium.

In addition, subcellular organelles, such as the sarcoplasmic reticulum, is known to be underdeveloped in immature myocardium, leading to greatly different cellular function from the mature myocardium. Immature myocardium has underdeveloped sarcoplasmic reticulum with lower calcium-storing ability, ${ }^{7}$ necessitating a larger extracellular calcium dependence ${ }^{8}$ in myocardial excitation-contraction coupling and intracellular calcium homeostasis, which is associated with greater involvement of calcium movement through the calcium channel compared to mature myocardium. It is obvious that immature myocardium may be largely different from mature myocardium from various viewpoints of cellular function, presumably affecting the composition when constructing a cardioplegic solution. St. Thomas' cardioplegic solution, a representative commercially available cardioplegic solution, can be used for adult cardiac surgery because the composition of this solution is generally recognized to have been determined based on the optimal cation concentration $\left(\mathrm{Na}^{+}, \mathrm{K}^{+}, \mathrm{Mg}^{2+}, \mathrm{Ca}^{2+}\right)$ obtained by a dose-response curve (i.e., relation between cation concentration and postischemic recovery) in an isolated working heart model using mature rat hearts. It may be questionable whether current cardioplegic solutions used for congenital heart surgery are appropriate for the immature myocardium because the composition of those solutions is almost the same as that of solutions for adult heart surgery. However, some say that current cardioplegic solutions for adult heart surgery can be used for congenital heart surgery. ${ }^{9}$

Although myocardial necrosis is still important, pathophysiology as a concept of cell death induced by ischemia and reperfusion and another concept, "apoptosis" (programmed cell death after various stimuli to the cell), have recently been investigated extensively in terms of mechanisms and prevention methods. Also, research on apoptosis after ischemia and reperfusion in the immature myocardium have recently gained focus, but investigation has not yet drawn concrete conclusions in terms of the underlying pathophysiology and its prevention. Karimi et al. observed more terminal dUTP nick-end labeling (TUNEL)-positive nuclei and a higher Bax/Bcl-2 ratio after cardioplegic arrest in newborn lamb hearts than in mature lamb hearts. They concluded that immature myocardium may exhibit greater apoptosis in response to ischemia and reperfusion than does mature myocardium. ${ }^{10}$

Fujii et al., using an isolated rat heart model with Krebs-Henseleit bicarbonate buffer, demonstrated that the hearts from cyanotic rats induced by a 6 -week duration of hypoxia from birth resulted in a greater postischemic recovery and a higher myocardial cyclic GMP level compared with the hearts from noncyanotic rats. They concluded that preischemic higher cyclic GMP levels in cyanotic myocardium may be related to enhanced tolerance to ischemia. The results of their study suggest altered energy metabolism in the cyanotic myocardium, and it is conceivable that cyanotic myocardium may result in enhanced anaerobic glycolysis in response to hypoxia, which may be associated with greater tolerance to ischemia.

Baker et al. have suggested that the myocardium from animals with chronic exposure to hypoxia from birth exhibits greater tolerance to ischemia, the mechanism of which may be related to cyclic GMP, nitric oxide production, and sarcolemmal $\mathrm{K}_{\text {ATP }}$ channel activation. ${ }^{11,12}$ However, Imura et al. investigated postischemic myocardial injury in pediatric patients who underwent cardiac surgery using crystalloid cardioplegia and suggested that postischemic myocardial injury might be dependent on the patient's age and the extent of cyanosis and the injury might be enhanced by more cyanosis. ${ }^{13}$ In support of this, an intriguing article from Wen et al. mentioned that expression of heat shock protein-70 in the postischemic myocardial cell was lower in patients with cyanotic heart disease than in those with noncyanotic heart disease. ${ }^{14}$ The results from these studies tell us there is controversy as to whether cyanosis exerts a cardioprotective effect against ischemia and reperfusion injury. The reason for the controversy about the cyanosis effect may be differences of experimental models (e.g., crystalloid or blood perfusion) or animal species, animals' age, and/or the presence or absence of hypoxia (or ischemia).

In conclusion, the article from Fujii et al. sends a significant message in that they elucidated that preischemic long-standing cyanosis may enhance tolerance to ischemia, although the results were obtained from the crystalloid perfusion model using isolated hearts from 6-week-old rats. Similar investigation using a heart immediately after birth and a blood perfusion model would be important for establishing myocardial protec- 
tion during neonatal or early infantile cardiac surgery. Careful manipulation of the heart is the most important fundamental procedure for myocardial protection regardless of whether it is an adult's or a child's heart. In fact, it is well to pay attention to the axiom, "the younger the patient, the more carefully you should manipulate the heart."

\section{References}

1. Doenst $\mathrm{T}$, Taegtmeyer $\mathrm{H}$. Ischemic preconditioning from bench to bedside. In: Beyersdorf $\mathrm{F}$, editor. Ischemiareperfusion in cardiac surgery. Austin: Landes; 2001. p. 108 24.

2. Rolph TP, Jones CT. Regulation of glycolytic flux in the heart of the fetal guinea pig. J Dev Physiol 1983;5:31-9.

3. Lopaschuk GD, Spafford MA, Marsh DR. Glycolysis is predominant source of ATP production immediately after birth. Am J Physiol 1991;261:H1698-705.

4. Grosso MA, Banerjee A, St. Cyr JA, Rogers KB, Brown JM, Clarke DR, et al. Cardiac 5'-nucleotidase activity increases with age and inversely relates to recovery from ischemia. J Thorac Cardiovasc Surg 1992;103:206-9.

5. Bolling SF, Olszanski DA, Bove EL, Childs KF. Enhanced myocardial protection during global ischemia with $5^{\prime}$ nucleotidase inhibitors. J Thorac Cardiovasc Surg 1992;103: 73-7.

6. Pridjian AK, Bove EL, Bolling SF, Childs KF, Brosamer KM, Lupinetti FM. Developmental differences in myocardial pro- tection in response to $5^{\prime}$-nucleotidase inhibition. J Thorac Cardiovasc Surg 1994;107:520-6.

7. Boucek RJ, Citak M, Braham TP, Artman M. Postnatal development of calcium release from cardiac sarcoplasmic reticulum. Pediatr Res 1984;18:119A.

8. Boland R, Martonosi M, Tillack TW. Developmental changes in the composition and function of sarcoplasmic reticulum. J Biol Chem 1974;249:612-23.

9. Doenst T, Schlensak C, Beyersdorf F. Cardioplegia in pediatric cardiac surgery: do we believe in magic? Ann Thorac Surg 2003;75:1668-77.

10. Karimi M, Wang LX, Hammel JM, Mascio CE, Abdulhamid $\mathrm{M}$, Barner EW, et al. Neonatal vulnerability to ischemia reperfusion: cardioplegic arrest causes greater myocardial apoptosis in neonatal than mature lambs. J Thorac Cardiovasc Surg 2004;127:490-7

11. Baker JE, Holman P, Kalyanaraman B, Griffith OW, Pritchard KA. Adaptation to chronic hypoxia confers tolerance to subsequent myocardial ischemia by increased nitric oxide production. Ann NY Acad Sci 1999;874:236-53.

12. Baker JE, Contney SJ, Singh R, Kalyanaraman B, Gross GJ, Bosnjak ZJ. Nitric oxide activate the sarcolemmal KATP channel in normothermic and chronically hypoxic hearts by a cyclic GMP-dependent mechanism. J Mol Cell Cardiol 2001;33:331-41.

13. Imura H, Caputo M, Parry A, Pawade A, Angelini GD, Suleiman MS. Age-dependent and hypoxia-related differences in myocardial protection during pediatric open heart surgery. Circulation 2001;103:1551-6.

14. Wen SB, Hu JG, Ma ZX, Zhou XM, Yang YF, Yu FL, et al. Expression of the heat shock protein-70 in the myocardial cells of cyanosis congenital heart diseases. Zhong Nan Da Xue Xue Bao Yi Xue Ban. 2004;29:322-5 (in Chinese). 